\title{
Metabolic syndrome prevalence according to ATP III and IDF criteria and related factors in Turkish adults
}

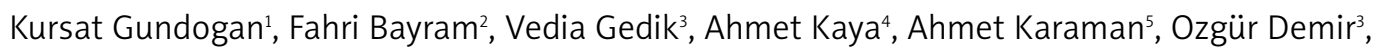
Tevfik Sabuncu ${ }^{6}$, Derya Kocer ${ }^{7}$, Ramazan Coskun ${ }^{1}$

1Department of Intensive Care, Faculty of Medicine, Erciyes University, Kayseri, Turkey 2Department of Endocrinology and Metabolism, Faculty of Medicine, Erciyes University, Kayseri, Turkey

${ }^{3}$ Department of Endocrinology and Metabolism, Faculty of Medicine, Ankara University, Ankara, Turkey

${ }^{4}$ Division of Endocrinology and Metabolism, Department of Internal Medicine, Meram Medical Faculty, Selcuk University, Konya, Turkey

${ }^{5}$ Department of Gastroenterology, Kayseri Research and Training Hospital, Kayseri, Turkey ${ }^{6}$ Department of Endocrinology and Metabolism, Faculty of Medicine, Harran University, Șanlıurfa, Turkey

7Department of Biochemistry, Kayseri Research and Training Hospital, Kayseri, Turkey

Submitted: 28 May 2012

Accepted: 29 August 2012

Arch Med Sci 2013; 9, 2: 243-253

DOI: 10.5114/aoms.2013.34560

Copyright (C 2013 Termedia \& Banach

\begin{abstract}
Introduction: The aim of this study is to investigate the prevalence of metabolic syndrome (MS) and its components according to Adult Treatment Panel III (ATP III) and International Diabetes Federation (IDF) criteria and the risk factors affecting MS. Metabolic syndrome prevalence was evaluated according to certain quintet age groups, altitude, location and demographic features.

Material and methods: This study was a cross-sectional survey conducted in 24 provinces from the 7 regions of Turkey. A total of 4309 adults from 7 regions participated in the study (1947 males, 45.2\%).

Results: The mean age of participants was $47 \pm 14$ years. Metabolic syndrome prevalence was found as $36.6 \%$ according to ATP III and $44.0 \%$ according to IDF. The MS rate was found to be higher in females compared to males in both groups $(p<0.01)$. According to both criteria, MS prevalence was found to be higher in subjects who lived in coastal regions when evaluated according to altitude and in subjects who lived in district centers when evaluated according to location. The MS risk is 1.62-fold higher in females compared to males. Metabolic syndrome risk increases as age increases and is highest in the 61-65 age group. Metabolic syndrome risk increases 2.75 -fold in the overweight compared to normal weighing subjects and 7.80-fold in the obese.

Conclusions: Metabolic syndrome prevalence was found to be high in Turkey according to both criteria. Metabolic syndrome prevalence increases as age and body mass index (BMI) increase. Age, female gender and obesity are independent risk factors for MS development.
\end{abstract}

Key words: metabolic syndrome, altitude, age groups.

\section{Introduction}

Metabolic syndrome (MS) consists of a cluster of several metabolic abnormalities, including hypertension, impaired glucose regulation, abdominal obesity and dyslipidemia [1, 2]. Other important characteris-

\author{
Corresponding author: \\ Kursat Gundogan MD \\ Erciyes University \\ Medical Faculty \\ 38039 Kayseri, Turkey \\ Phone: 5053166107 \\ E-mail: kursatgundogan@ \\ hotmail.com
}


tics of MS include low-grade inflammation, endothelial dysfunction, plasma hypercoagulability and atherosclerosis [3]. Metabolic syndrome has been demonstrated as a common precursor to the development of diabetes mellitus (DM) and cardiovascular disease (CVD) and individuals with MS are associated with approximately five and twofold increased risk for DM and CVD respectively [4]. Metabolic syndrome is a risk factor for all-cause mortality [5]. It has also been linked with obesity and a sedentary lifestyle, both of which are modifiable [6].

The prevalence of MS varies greatly between countries and ethnic groups [7]. It is associated with life-style, demographic, socio-economic, and genetic factors. Age, body mass index, postmenopausal status, a diet rich in saturated fats, carbohydrates, and smoking have been positively associated with MS, while inverse associations have been shown for physical activity, education, income, and alcohol intake [8-10].

The prevalence of metabolic syndrome worldwide is in the range $7.9-43 \%$ and $7-56 \%$ in males and in females, respectively [11-13]. The prevalence of metabolic syndrome was reported to vary from $23.7 \%$ to $32.2 \%$ in males and $38.6 \%$ to $45.0 \%$ in females in previous studies conducted in different parts of Turkey [14-18].

The aim of this study is to investigate the prevalence of MS and its components among Turkish adults according to ATP III and IDF criteria and to examine the difference from others in that it assesses risk factors affecting MS according to demographic features. Our study aimed to reveal MS incidence and the risk factors affecting MS according to quintet age groups, altitude and site (city center, district, village); these factors were not taken into consideration or investigated in previous studies.

\section{Material and methods}

\section{Study plan and sampling}

This study was a cross-sectional survey. The sampling design used was multistage probability sampling. Approval was obtained from the Ethical Committee of the Ministry of Health and the household identification form (HIF) data were obtained from the Primary Health Care Centers of the Provincial Health Directorates affiliated to the Ministry of Health.

This study was conducted in 24 provinces (Adana, Ankara, Antalya, Bursa, Çanakkale, Denizli, Diyarbakır, Edirne, Erzurum, Eskișehir, Gaziantep, Giresun, Hatay, İstanbul, İzmir, Mersin, Kars, Kayseri, Konya, Samsun, Sivas, Șanlıurfa, Van, Zonguldak) from the 7 regions of Turkey (Figure 1). At least 3 provinces were selected from each region by a random sampling method. The populations of these 7 regions were obtained from the records of the 2000 census. The study sample included males and nonpregnant females aged between 20 and 83 years. The populations of city centers, districts, and villages were classified by using the stratified sampling method and then were selected from the HIF data by a random sampling method. The geography of Turkey was classified into three groups according to altitude. Sea level was accepted as zero. 0-300 $\mathrm{m}$ was taken as coastal, $300-900 \mathrm{~m}$ as moderate elevation and $900 \mathrm{~m}$ and above as high elevation.

The age groups were also classified according to the records of the 2000 census as follows: $20-25$ years, 26-30 years, 31-35 years, 36-40 years, $41-45$ years, $46-50$ years, $51-55$ years, $56-60$ years, $61-65$ years, 66-70 years, and 70 years and above. This classification was adapted to reflect the characteristics of Turkey. Characteristics of the provincial district centers and villages, including gender, demo-

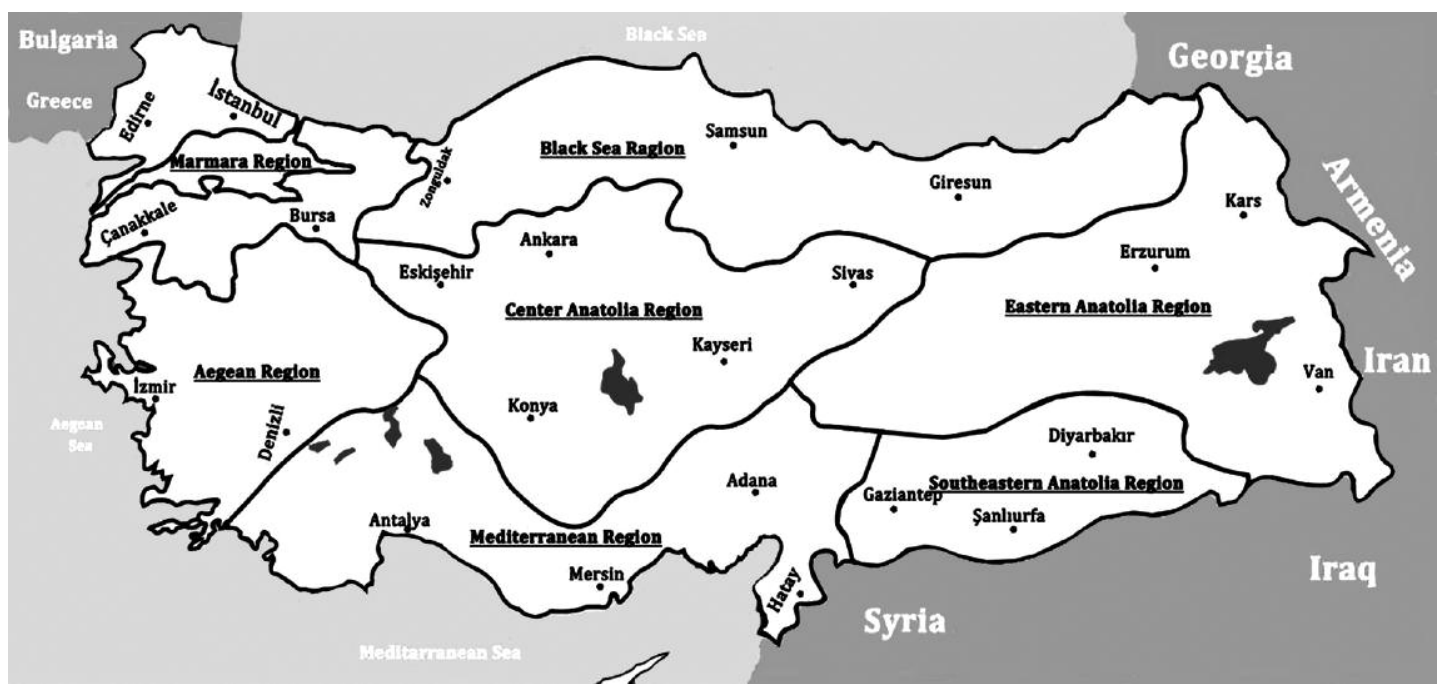

Figure 1. Map of Turkey 
graphics, economic, social, and geographical status, were taken into consideration.

Medical establishments were informed (primary health-care centers, hospitals, and local health clinics) about the research 2 weeks before the study. The participants were asked to fast for 12-16 h prior to the sampling. Between 7:00 and 10:00 a.m., about 100 people were evaluated in health units affiliated to the Ministry of Health. Comfortable waiting rooms were provided for the researchers during the study. Informed consent was obtained from all participants. Patients' informed consents were obtained. Structured questionnaires were completed with face-to-face interviews. The study was employed in accordance with the Declaration of Helsinki.

\section{Study protocol}

The medical histories and measurements of the participants were obtained by well-trained nurses and specialist physicians. The ages and genders of the participants were recorded. Personal and family histories of hypertension, diabetes mellitus, cardiovascular diseases, and other chronic diseases were obtained. Heights and weights of participants were measured. When the subjects were weighed, they were asked to take off their shoes and any other belongings that could possibly add extra weight. Heights and weights were evaluated according to body mass index (BMI). Body mass index was calculated by dividing the weight (in $\mathrm{kg}$ ) by the height in meters squared. Body mass index below $19 \mathrm{~kg} / \mathrm{m}^{2}$ was classified as underweight and one between $19 \mathrm{~kg} / \mathrm{m}^{2}$ and $25 \mathrm{~kg} / \mathrm{m}^{2}$ as normal; BMI values between $25 \mathrm{~kg} / \mathrm{m}^{2}$ and $29.9 \mathrm{~kg} / \mathrm{m}^{2}$ were considered as overweight, above $30 \mathrm{~kg} / \mathrm{m}^{2}$ as obese and $40 \mathrm{~kg} / \mathrm{m}^{2}$ as morbidly obese. Also the waist circumference (WC) of participants was measured at the level of the iliac processes and the umbilicus with a soft tape measure to evaluate abdominal obesity. Systolic blood pressure (SBP) and diastolic blood pressure (DBP) were measured twice in the sitting position, with an interval of $15 \mathrm{~min}$ between the measurements, by means of standard sphygmomanometers of appropriate width, after a rest period for $30 \mathrm{~min}$. The limit for systolic hypertension was $130 \mathrm{~mm} \mathrm{Hg}$ and for diastolic hypertension was $85 \mathrm{~mm} \mathrm{Hg}$. Those taking antihypertensive therapy were considered to be hypertensive even if their blood pressure was not above $130 / 85 \mathrm{~mm} \mathrm{Hg}$.

Blood samples were centrifuged at room temperature for $10 \mathrm{~min}$ at $3000 \mathrm{rpm}$. The extracted sera were stored in ice bags and placed into deep freezers at $-70^{\circ} \mathrm{C}$ on the same day. Glucose, total cholesterol, high-density lipoprotein cholesterol (HDL-C) and triglyceride (TG) levels were evaluated by the enzymatic spectrophotometric method with the
Kone Lab Auto Analyzer (Thermo Clinical Labsystems Oy Vantaa, Finland). Low-density lipoprotein cholesterol (LDL-C) was calculated by the Friedewald formula (in those with a triglyceride level below $400 \mathrm{mg} / \mathrm{dl}$ ). Diabetes mellitus was diagnosed according to the American Diabetes Association (ADA) criteria. Accordingly, single fasting blood glucose of above $126 \mathrm{mg} / \mathrm{dl}$ was considered to be evidence of diabetes. Those with a previous diabetes diagnosis and using oral antidiabetics and/or insulin were also considered as diabetics. Impaired fasting glucose was defined as fasting blood glucose levels between $110 \mathrm{mg} / \mathrm{dl}$ and $126 \mathrm{mg} / \mathrm{dl}$. The diagnosis of metabolic syndrome was made depending on the presence of at least 3 of the following parameters, according to Adult Treatment Panel III-2001 (ATP III) criteria: abdominal obesity (WC > $102 \mathrm{~cm}$ for males and $>88 \mathrm{~cm}$ for females), hypertension (SBP > $130 \mathrm{~mm} \mathrm{Hg}$ and/or DBP > $85 \mathrm{~mm} \mathrm{Hg}$ ) or history of antihypertensive usage, hypertriglyceridemia $(\geq 150 \mathrm{mg} / \mathrm{dl}$ ) or presence of treatment for this disorder, low HDL-C (< $40 \mathrm{mg} / \mathrm{dl}$ in males and < $50 \mathrm{mg}$ / $\mathrm{dl}$ in females), and high fasting plasma glucose ( $\geq 110 \mathrm{mg} / \mathrm{dl}$ ) or presence of diagnosis of type 2 diabetes mellitus (T2DM) [19].

Metabolic syndrome prevalence was also calculated according to IDF criteria: abdominal obesity (WC $>94 \mathrm{~cm}$ for males and $>80 \mathrm{~cm}$ for females) has to be present and at least 2 of the following 4 parameters should be present: hypertension (SBP $>130 \mathrm{~mm} \mathrm{Hg}$ and/or DBP > $85 \mathrm{~mm} \mathrm{Hg}$ ) or history of antihypertensive usage, hypertriglyceridemia $(\geq 150 \mathrm{mg} / \mathrm{dl})$ or presence of treatment for this disorder, low HDL-C ( $<40 \mathrm{mg} / \mathrm{dl}$ in males and $<50 \mathrm{mg} / \mathrm{dl}$ in females) or presence of treatment for this disorder, and high fasting plasma glucose (> $100 \mathrm{mg} / \mathrm{dl}$ ) or presence of diagnosis of T2DM [1].

\section{Statistical analysis}

The $\chi^{2}$ test was used to determine significant differences in proportions among categorical variables. The Student $t$-test and Mann-Whitney $U$-test were used to compare differences of continuous variables. The univariate and multiple (model: backward Wald) binary logistic regression models were used to investigate the probability of having metabolic syndrome according to sociodemographic variables. Odds ratios (OR) and 95\% confidence intervals $(\mathrm{Cl})$ were calculated using binary logistic regression for each model. All statistical analyses were calculated using SPSS version 15.0 (Chicago, IL). Two-tailed values of $p$ of $<0.05$ were considered to be statistically significant.

\section{Results}

A total of 4309 adults from 7 regions participated in the study (1947 males, 45.2\%; 2362 females, 
Table I. Demographic characteristics of participants with and without metabolic syndrome

\begin{tabular}{|lccccccc|}
\hline Variables & $\begin{array}{c}\text { General } \\
(n=4307)\end{array}$ & \multicolumn{2}{c}{ Gender } & \multicolumn{2}{c|}{ Metabolic syndrome (ATP III) } & Metabolic syndrome (IDF) \\
\cline { 3 - 7 } & & $\begin{array}{c}\text { Male } \\
(n=1947)\end{array}$ & $\begin{array}{c}\text { Female } \\
(n=2362)\end{array}$ & $\begin{array}{c}\text { Yes } \\
(n=1577)\end{array}$ & $\begin{array}{c}\text { No } \\
(n=2732)\end{array}$ & $\begin{array}{c}\text { Yes } \\
(n=1896)\end{array}$ & $\begin{array}{c}\text { No } \\
(n=2413)\end{array}$ \\
\hline Age [years] & $47.1 \pm 14.7$ & $49.1 \pm 14.6^{\mathrm{a}}$ & $45.5 \pm 14.8$ & $51.8 \pm 13.1^{\mathrm{a}}$ & $44.4 \pm 15.0$ & $51.6 \pm 13.0^{\mathrm{a}}$ & $43.6 \pm 15.1$ \\
\hline SBP [mm Hg] & $133.5 \pm 27.2$ & $133.1 \pm 26.6^{\mathrm{d}}$ & $133.9 \pm 27.8$ & $145.2 \pm 25.9^{\mathrm{a}}$ & $126.9 \pm 25.7$ & $144.0 \pm 26.3^{\mathrm{a}}$ & $125.3 \pm 25.0$ \\
\hline DBP [mm Hg] & $81.4 \pm 15.1$ & $81.2 \pm 15.3^{\mathrm{d}}$ & $81.6 \pm 14.9$ & $86.6 \pm 14.1^{\mathrm{a}}$ & $78.4 \pm 14.9$ & $86.2 \pm 14.3^{\mathrm{a}}$ & $77.6 \pm 14.6$ \\
\hline BMl [kg/m²] & $28.3 \pm 5.2$ & $27.4 \pm 4.40$ & $29.16 \pm 5.70^{\mathrm{a}}$ & $31.2 \pm 5.0^{\mathrm{a}}$ & $26.7 \pm 4.6$ & $31.1 \pm 4.7^{\mathrm{a}}$ & $26.2 \pm 4.6$ \\
\hline WC [cm] & $92.6 \pm 12.9$ & $95.6 \pm 11.9^{\mathrm{a}}$ & $90.2 \pm 13.2$ & $100.1 \pm 10.8^{\mathrm{a}}$ & $88.3 \pm 12.0$ & $100.1 \pm 9.9^{\mathrm{a}}$ & $86.9 \pm 12.1$ \\
\hline $\begin{array}{l}\text { Fasting blood } \\
\text { glucose [mg/dl] }\end{array}$ & $103.7 \pm 44.8$ & $102.8 \pm 41.7^{\mathrm{d}}$ & $104.6 \pm 47.3$ & $122.8 \pm 61.6^{\mathrm{a}}$ & $92.8 \pm 25.5$ & $117.2 \pm 55.8^{\mathrm{a}}$ & $93.1 \pm 29.9$ \\
\hline $\begin{array}{l}\text { Triglycerides } \\
\text { [mg/dl] }\end{array}$ & $145.4 \pm 96.3$ & $152.7 \pm 103.3^{\mathrm{a}}$ & $139.5 \pm 89.7$ & $201.7 \pm 113.4^{\mathrm{a}}$ & $113.0 \pm 65.9$ & $185.7 \pm 110.3^{\mathrm{a}}$ & $113.8 \pm 68.9$ \\
\hline $\begin{array}{l}\text { Total cholesterol } \\
\text { [mg/dl] }\end{array}$ & $194.2 \pm 47.7$ & $191.2 \pm 47.5$ & $196.8 \pm 47.8^{\mathrm{a}}$ & $208.4 \pm 48.4^{\mathrm{a}}$ & $186.1 \pm 45.4$ & $206.4 \pm 48.7^{\mathrm{a}}$ & $184.7 \pm 44.7$ \\
\hline HDL-C [mg/dl] & $50.3 \pm 16.3$ & $47.0 \pm 15.9$ & $53.1 \pm 16.3^{\mathrm{a}}$ & $44.3 \pm 14.1^{\mathrm{a}}$ & $53.8 \pm 16.4$ & $46.2 \pm 15.9^{\mathrm{a}}$ & $53.5 \pm 16.0$ \\
\hline LDL-C [mg/dl] & $117.7 \pm 41.0$ & $117.1 \pm 40.9^{\mathrm{d}}$ & $118.3 \pm 41.2$ & $126.9 \pm 41.6^{\mathrm{a}}$ & $112.6 \pm 39.8$ & $126.7 \pm 42.0^{\mathrm{a}}$ & $110.9 \pm 38.9$ \\
\hline
\end{tabular}

$a_{p}<0.001, b_{p}<0.01, c_{p}<0.05, d_{p}>0.05$

54.8\%): Marmara Region, 820 (19.0\%); Central Anatolian Region, 816 (18.9\%); Aegean Region, 424 (9.8\%); Mediterranean Region, 674 (15.6\%); Black Sea Region, 535 (12.4\%); Southeast Region, 437 (10.1\%); Eastern Region, 603 (14.0\%). The demographic characteristics of the participants who were and were not diagnosed with metabolic syndrome are shown in Table I.

The mean age of participants was $47.1 \pm 14.7$ years $(20-83)=49.0 \pm 14.5$ for males, $45.5 \pm 14.7$ for females. Distribution of participants according to age groups was as follows: $7.4 \%(n=319)$ between 20 and 25 years, $6.8 \%(n=294)$ between 26 and 30 years, $10.0 \%(n=433)$ between 31 and 35 years, $12.0 \%(n=519)$ between 36 and 40 years, $11.4 \%$ $(n=492)$ between 41 and 45 years, $11.6 \%(n=498)$ between 46 and 50 years, $11.3 \%(n=487)$ between 51 and 55 years, 9.0\% $(n=387)$ between 56 and 60 years, $7.3 \%(n=314)$ between 61 and 65 years, $6.3 \%(n=273)$ between 66 and 70 years, and $6.8 \%$ $(n=293)$ over 70 years.

The locations of participants were as follows: $40.1 \%(n=1729)$ lived in city centers, 36.9\% $(n=1589)$ in districts and $23 \%(n=991)$ in villages.

The distribution of participants according to altitude was as follows: coast $48.2 \%(n=2079)$, moderate elevation $22.8 \%(n=977)$ and high elevation $29.1 \%(n=1253)$.

Metabolic syndrome prevalence was found to be 36.6\% (males: $30.3 \%$, females: $41.8 \%$ ) according to ATP III criteria and $44.0 \%$ according to IDF criteria (males: $37.0 \%$, females: $49.8 \%$ ) (Table II). The MS rate was found to be higher in females compared to males in both groups $(p<0.01)$.

Metabolic syndrome prevalence was assessed according to gender, age groups, BMI, regions, loca- tion and altitude by using ATP III and IDF criteria (Table II).

Metabolic syndrome incidence was found to be $79 \%$ among diabetics and $52.3 \%$ among hypertensives in all participants according to ATP III criteria.

Prevalence of MS components was assessed according to gender, age groups, BMI, regions, location and altitude by using ATP III and IDF criteria (Tables III and IV). Hypertension was found to be the highest component according to both ATP III and IDF criteria (Table V). Univariate and multiple logistic regression analyses are shown in Table VI. Metabolic syndrome prevalence of provinces according to ATP III and IDF criteria is shown in Table VII.

\section{Discussion}

This study was conducted with the aim of investigating the prevalence of MS and its components in Turkish adults according to ATP III and IDF criteria. It differs from the other studies conducted in Turkey in many aspects. This study reflects Turkey in a comprehensive manner in terms of demographic and socio-economic features, includes females and males in quintet age groups, detects affecting risk factors, makes assessments according to locations (city center, district and village) and evaluates MS according to altitude, a factor which has never been studied before.

No studies are available including the seven regions of Turkey and demographic and socio-economic features. In local studies conducted in our country, MS prevalence was found as $21.7-32.2 \%$ in males and $31.3-45.0 \%$ in females according to ATP III criteria [14-16, 20]. As seen in the aforementioned reports, MS prevalence was detected to be higher 
Table II. Absence or presence of metabolic syndrome

\begin{tabular}{|c|c|c|c|c|}
\hline \multirow[t]{2}{*}{ Variants } & \multicolumn{2}{|c|}{ Metabolic syndrome (ATP III) } & \multicolumn{2}{|c|}{ Metabolic syndrome (IDF) } \\
\hline & Present, $n(\%)$ & Absent, $n(\%)$ & Present, $n(\%)$ & Absent, $n(\%)$ \\
\hline \multicolumn{5}{|l|}{ Gender } \\
\hline Male & $589(30.3)$ & $1358(69.7)$ & $720(37)$ & $1227(63)$ \\
\hline Female & $988(41.8)^{a}$ & $1374(50.3)$ & $1176(49.8)^{\mathrm{a}}$ & $1186(50.2)$ \\
\hline \multicolumn{5}{|l|}{ Age groups [years] } \\
\hline $18-25$ & $21(6.6)$ & $298(93.4)$ & $24(7.5)$ & $295(92.5)$ \\
\hline $26-30$ & $52(17.7)$ & $242(82.3)$ & $68(23.1)$ & $226(76.9)$ \\
\hline $31-35$ & $124(28.6)$ & $309(71.4)$ & $148(34.2)$ & $285(65.8)$ \\
\hline $36-40$ & $160(30.8)$ & $359(69.2)$ & $193(37.2)$ & $326(62.8)$ \\
\hline $41-45$ & $157(31.9)$ & $335(68.1)$ & $200(40.7)$ & $292(59.7)$ \\
\hline $46-50$ & $199(40)$ & $299(60)$ & $233(46.8)$ & $265(53.2)$ \\
\hline $51-55$ & $247(50.7)$ & $240(49.3)$ & $296(60.8)^{a}$ & $191(39.2)$ \\
\hline $56-60$ & $196(50.6)$ & $191(49.4)$ & $235(60.7)$ & $152(39.3)$ \\
\hline $61-65$ & $160(51)^{\mathrm{a}}$ & $154(49)$ & $185(58.9)$ & $129(41.1)$ \\
\hline $66-70$ & $133(48.7)$ & $140(51.3)$ & $162(59.3)$ & $111(40.7)$ \\
\hline$\geq 71$ & $128(43.7)$ & $165(56.3)$ & $152(51.9)$ & $141(48.1)$ \\
\hline \multicolumn{5}{|l|}{ BMI } \\
\hline Normal & $139(12.8)$ & $947(87.2)$ & $129(11.9)$ & $957(88.1)$ \\
\hline Overweight & $516(32.3)$ & $1083(67.7)$ & $687(43.0)$ & $912(57)$ \\
\hline Obese & $843(57.8)$ & $615(42.2)$ & $996(68.3)$ & $462(31.7)$ \\
\hline Morbidly obese & $76(79.2)^{a}$ & $20(20.8)$ & $80(83.3)^{a}$ & $16(16.7)$ \\
\hline \multicolumn{5}{|l|}{ Regions } \\
\hline Mediterranean Region & $233(34.6)$ & $441(65.4)$ & $289(42.9)$ & $385(57.1)$ \\
\hline Central Anatolian Region & $348(42.6)$ & $468(57.4)$ & $410(52.9)$ & $406(49.8)$ \\
\hline Black Sea Region & $152(28.4)$ & $383(71.6)$ & $195(36.4)$ & $340(63.6)$ \\
\hline Aegean Region & $170(40.1)$ & $254(59.9)$ & $205(48.3)$ & $219(51.7)$ \\
\hline Marmara Region & $398(48.5)^{a}$ & $422(51.5)$ & $475(57.9)^{a}$ & $345(42.9)$ \\
\hline Southeast Region & $155(25.7)$ & $448(74.3)$ & $180(29.9)$ & $423(70.1)$ \\
\hline Eastern Region & $121(27.7)$ & $316(72.3)$ & $142(32.5)$ & $295(67.5)$ \\
\hline \multicolumn{5}{|l|}{ Location } \\
\hline City center & $640(37)$ & $1089(63)$ & $756(43.7)$ & $973(56.3)$ \\
\hline District center & $616(38.8)^{c}$ & $973(61.2)$ & $739(46.5)^{c}$ & $850(53.5)$ \\
\hline Village & $321(32.4)$ & $670(67.6)$ & $401(40.5)$ & $590(59.5)$ \\
\hline \multicolumn{5}{|l|}{ Altitude } \\
\hline Coastal & $830(39.9)^{a}$ & $1249(60.1)$ & $996(47.9)^{a}$ & $1083(52.1)$ \\
\hline Moderate elevation & $278(28.5)$ & $699(71.5)$ & 348 (35.6) & $629(64.4)$ \\
\hline High elevation & $469(37.4)$ & $784(62.6)$ & $552(44.1)$ & $701(55.9)$ \\
\hline
\end{tabular}

$a_{p}<0.001, b_{p}<0.01, c_{p}<0.05, d_{p}>0.05$

in females compared to males. The high prevalence of MS in females may be due to high abdominal obesity rates in females. In two studies from Turkey, when subjects were evaluated according to denary age groups, MS prevalence was found to be highest in the 60-69 age group $[14,20]$. Our assessment was done according to quintet age groups and MS prevalence was similarly found to be highest in the 61-65 age group. Metabolic syndrome prevalence increases as age increases, but it tends to decrease especially after 70 [20-22]. This may be related to increasing chronic diseases and their complications, 
Table III. Prevalence of metabolic syndrome components in the study population (ATP III)

\begin{tabular}{|c|c|c|c|c|c|}
\hline Variants & $\begin{array}{c}\text { Hypertension } \\
n(\%)\end{array}$ & $\begin{array}{c}\text { Hyperglycemia } \\
n(\%)\end{array}$ & $\begin{array}{c}\text { Abdominal } \\
\text { obesity, } n(\%)\end{array}$ & $\begin{array}{l}\text { Hypertriglyceridemia } \\
n(\%)\end{array}$ & $\begin{array}{l}\text { Low HDL-C } \\
\text { levels, } n(\%)\end{array}$ \\
\hline \multicolumn{6}{|l|}{ Gender } \\
\hline Male & $1114(57.2)^{d}$ & $482(24.8)^{d}$ & $530(27.2)$ & $746(38.3)^{b}$ & 699 (35.9) \\
\hline Female & $1388(58.8)$ & $577(24.4)$ & $1328(56.2)^{a}$ & 791 (33.5) & $1091(46.2)^{a}$ \\
\hline \multicolumn{6}{|l|}{ Age groups [years] } \\
\hline $18-25$ & $85(26.6)$ & $24(7.5)$ & $30(9.4)$ & $49(15.4)$ & $118(37.0)$ \\
\hline $26-30$ & $97(33.0)$ & 31 (10.5) & $70(23.8)$ & $74(25.2)$ & $124(42.2)$ \\
\hline $31-35$ & $185(42.7)$ & $64(14.8)$ & $154(35.6)$ & $135(31.2)$ & $186(43.0)$ \\
\hline $36-40$ & 219 (42.2) & 83 (16.0) & 206 (39.7) & 193 (37.2) & $238(45.9)^{c}$ \\
\hline $41-45$ & $242(49.2)$ & 105 (21.3) & 217 (44.1) & 188 (38.2) & $204(41.5)$ \\
\hline $46-50$ & $300(60.2)$ & $134(26.9)$ & $237(47.6)$ & 207 (41.6) & $202(40.6)$ \\
\hline $51-55$ & 349 (71.7) & $157(32.2)$ & $271(55.6)^{a}$ & $235(48.3)^{a}$ & $212(43.5)$ \\
\hline $56-60$ & $300(77.5)$ & 139 (35.9) & $215(55.6)$ & $161(41.6)$ & $163(42.1)$ \\
\hline $61-65$ & $252(80.3)$ & 109 (34.7) & $174(55.4)$ & $121(38.5)$ & $140(44.6)$ \\
\hline $66-70$ & $229(83.9)^{c}$ & $103(37.7)^{a}$ & $147(53.8)$ & $84(30.8)$ & 108 (39.6) \\
\hline$\geq 71$ & $244(83.3)$ & $110(37.5)$ & $137(46.8)$ & $90(30.7)$ & $95(32.4)$ \\
\hline \multicolumn{6}{|l|}{ BMI } \\
\hline Normal & $463(40.1)$ & 171 (14.8) & $72(6.2)$ & 239 (20.7) & 406 (35.1) \\
\hline Overweight & $952(59.5)$ & $373(23.3)$ & $543(34.0)$ & $589(36.8)$ & $665(41.6)$ \\
\hline Obese & 1012 (69.4) & $469(32.2)$ & $1149(78.8)$ & $659(45.2)$ & $664(45.5)$ \\
\hline Morbidly obese & $75(78.1)^{a}$ & $46(47.9)^{a}$ & $94(97.9)^{a}$ & $50(52.1)^{a}$ & $55(57.3)^{a}$ \\
\hline \multicolumn{6}{|l|}{ Regions } \\
\hline Mediterranean Region & $437(64.8)$ & $143(21.2)$ & $321(47.6)$ & $295(43.8)^{a}$ & $159(23.6)$ \\
\hline Central Anatolian Region & $560(68.6)^{a}$ & $210(25.7)$ & $386(47.3)$ & $310(38.0)$ & $373(45.7)$ \\
\hline Black Sea Region & $345(64.5)$ & $120(22.4)$ & 209 (39.1) & $165(30.8)$ & $139(26.0)$ \\
\hline Aegean Region & $193(45.5)$ & $124(29.2)$ & $196(46.2)$ & $137(32.3)$ & $216(50.9)$ \\
\hline Marmara Region & $501(61.1)$ & $293(35.7)^{a}$ & $453(55.2)^{a}$ & $308(37.6)$ & $430(52.4)^{a}$ \\
\hline Southeast Region & $215(35.7)$ & $98(16.3)$ & $150(24.9)$ & $204(33.8)$ & $311(51.6)$ \\
\hline Eastern Region & $251(57.4)$ & $71(16.2)$ & $143(32.7)$ & $118(27.0)$ & $162(37.1)$ \\
\hline \multicolumn{6}{|l|}{ Location } \\
\hline City center & $903(52.2)$ & $452(26.1)^{b}$ & $748(43.3)^{d}$ & $656(37.9)^{c}$ & $724(41.9)$ \\
\hline District center & $952(59.9)$ & $399(25.1)$ & $692(43.5)$ & $558(35.1)$ & $735(46.3)^{a}$ \\
\hline Village & $647(65.3)^{a}$ & $208(21.0)$ & $418(42.2)$ & $323(32.6)$ & 331 (33.4) \\
\hline \multicolumn{6}{|l|}{ Altitude } \\
\hline Coastal & $1270(61.1)$ & $562(27.0)^{b}$ & $1024(49.3)^{a}$ & $787(37.9)^{c}$ & $812(39.1)$ \\
\hline Moderate elevation & $421(43.1)$ & $216(22.1)$ & 305 (31.29) & $322(33.0)$ & $443(45.3)^{b}$ \\
\hline High elevation & $811(64.7)^{\mathrm{a}}$ & $281(22.4)$ & $529(42.2)$ & $428(34.2)$ & $535(42.7)$ \\
\hline
\end{tabular}

$a_{p}<0.001, b_{p}<0.01, c_{p}<0.05, d_{p}>0.05$

such as malnutrition, dementia, etc, that increase with age. In one of our previous studies conducted in the Mediterranean region of Turkey, MS prevalence was found to be $43.2 \%$ in the obese population and $32.7 \%$ in provincials [14]. Similarly, in this study it was found to be highest in the obese
(57.8\%) and morbidly obese (79.2\%), in districts (38.8\%). Metabolic syndrome prevalence was found to be higher among individuals who live on the coast according to both ATP III and IDF criteria. We could not find any study in the literature on this topic. The higher rate found on the coast may be 
Table IV. Prevalence of metabolic syndrome components in the study population (IDF)

\begin{tabular}{|c|c|c|c|c|}
\hline Variants & $\begin{array}{c}\text { Hypertension } \\
n(\%)\end{array}$ & $\begin{array}{l}\text { Hyperglycemia } \\
n(\%)\end{array}$ & $\begin{array}{l}\text { Hypertriglyceridemia } \\
n(\%)\end{array}$ & $\begin{array}{l}\text { Low HDL-C } \\
\text { levels, } n(\%)\end{array}$ \\
\hline \multicolumn{5}{|l|}{ Gender } \\
\hline Male & $1114(57.2)^{d}$ & $723(37.1)^{d}$ & $746(38.3)^{b}$ & 699 (35.9) \\
\hline Female & $1388(58.8)$ & $871(36.9)$ & $791(33.5)$ & $1091(46.2)^{a}$ \\
\hline \multicolumn{5}{|l|}{ Age groups [years] } \\
\hline $18-25$ & $85(26.6)$ & $47(14.7)$ & $49(15.4)$ & $118(37.0)$ \\
\hline $26-30$ & $97(33.0)$ & $62(21.1)$ & $74(25.2)$ & $124(42.2)$ \\
\hline $31-35$ & $185(42.7)$ & $119(27.5)$ & $135(31.2)$ & $186(43.0)$ \\
\hline $36-40$ & $219(42.2)$ & $142(27.4)$ & $193(37.2)$ & $238(45.9)^{c}$ \\
\hline $41-45$ & $242(49.2)$ & $169(34.3)$ & $188(38.2)$ & $204(41.5)$ \\
\hline $46-50$ & $300(60.2)$ & $209(42.0)$ & 207 (41.6) & $202(40.6)$ \\
\hline $51-55$ & $349(71.7)$ & $226(46.4)$ & $235(48.3)^{a}$ & $212(43.5)$ \\
\hline $56-60$ & $300(77.5)$ & $188(48.5)$ & $161(41.6)$ & $163(42.1)$ \\
\hline $61-65$ & $252(80.3)$ & $159(50.6)^{a}$ & $121(38.5)$ & $140(44.6)$ \\
\hline $66-70$ & $229(83.9) c$ & $133(48.7)$ & $84(30.8)$ & $108(39.6)$ \\
\hline$\geq 71$ & $244(83.3)$ & $140(47.8)$ & $90(30.7)$ & $95(32.4)$ \\
\hline \multicolumn{5}{|l|}{ BMI } \\
\hline Normal & $463(40.1)$ & $298(25.8)$ & $239(20.7)$ & $406(35.1)$ \\
\hline Overweight & $952(59.5)$ & $552(34.5)$ & $589(36.8)$ & $665(41.6)$ \\
\hline Obese & $1012(69.4)$ & $687(47.1)$ & $659(45.2)$ & $664(45.5)$ \\
\hline Morbidly obese & $75(78.1)^{a}$ & $57(59.4)^{a}$ & $50(52.1)^{\mathrm{a}}$ & $55(57.3)^{a}$ \\
\hline \multicolumn{5}{|l|}{ Regions } \\
\hline Mediterranean Region & $437(64.8)$ & $216(32.0)$ & $295(43.8)^{a}$ & $159(23.6)$ \\
\hline Central Anatolian Region & $560(68.6)^{\mathrm{a}}$ & $278(34.1)$ & $310(38.0)$ & $373(45.7)$ \\
\hline Black Sea Region & $345(64.5)$ & $182(34.0)$ & $165(30.8)$ & $139(26.0)$ \\
\hline Aegean Region & $193(45.5)$ & $201(47.4)$ & $137(32.3)$ & $216(50.9)$ \\
\hline Marmara Region & $501(61.1)$ & $440(53.7)^{a}$ & $308(37.6)$ & $430(52.4)^{a}$ \\
\hline Southeast Region & $215(35.7)$ & $171(28.4)$ & $204(33.8)$ & $311(51.6)$ \\
\hline Eastern Region & $251(57.4)$ & $106(24.3)$ & $118(27.0)$ & $62(37.1)$ \\
\hline \multicolumn{5}{|l|}{ Location } \\
\hline City center & $903(52.2)$ & $690(39.9)^{a}$ & $656(37.9)^{c}$ & $724(41.9)$ \\
\hline District center & $952(59.9)$ & $598(37.6)$ & $558(35.1)$ & $735(46.3)^{a}$ \\
\hline Village & $647(65.3)^{\mathrm{a}}$ & $306(30.9)$ & $323(32.6)$ & $331(33.4)$ \\
\hline \multicolumn{5}{|l|}{ Altitude } \\
\hline Coastal & $1270(61.1)$ & $835(40.2)^{a}$ & $787(37.9)^{c}$ & $812(39.1)$ \\
\hline Moderate elevation & $421(43.1)$ & $375(38.4)$ & $322(33.0)$ & $443(45.3)^{b}$ \\
\hline High elevation & $811(64.7)^{a}$ & $384(30.6)$ & $428(34.2)$ & $535(42.7)$ \\
\hline
\end{tabular}

Abdominal obesity has to be present in IDF criteria. ${ }^{a} p<0.001, b_{p}<0.01, c_{p}<0.05, d_{p}>0.05$

due to higher socio-economic levels, nutrition type, sedentary life style and higher mean annual temperature.

In a study conducted in Greece, which is a neighbor of Turkey and has the same geographic and demographic features as Turkey, overall MS prevalence was detected as $23.6 \%$ (24.2\% for males, $22.8 \%$ for females) [23]. In contrast to our study, MS prevalence was found to be higher in males. While living areas were classified as urban and rural areas in other studies, they were classified as urban, semi-urban and rural areas as in this 
Table V. Prevalence of metabolic syndrome components in the population

\begin{tabular}{|lcc|}
\hline Components & $\begin{array}{c}\text { Metabolic } \\
\text { syndrome } \\
\text { ATP III }(n=1577) \\
n(\%)\end{array}$ & $\begin{array}{c}\text { Metabolic } \\
\text { syndrome } \\
\text { IDF }(n=1896) \\
n(\%)\end{array}$ \\
\hline Hypertension & $1380(87.5)$ & $1592(84.0)$ \\
\hline Hyperglycemia, IGF, DM & $811(51.4)$ & $1131(59.7)$ \\
\hline Abdominal obesity & $1217(77.2)$ & $1896(100)^{a}$ \\
\hline Hypertriglyceridemia & $1098(69.9)$ & $1124(59.3)$ \\
\hline Low HDL-C & $1078(68.4)$ & $1150(60.7)$ \\
\hline aAbdominal obesity has to be present in IDF criteria &
\end{tabular}

study. Obesity, HT and DM were found to be higher in subjects living in urban areas. Similarly to the studies above, in our study, MS prevalence was found to be significantly higher in individuals liv- ing in districts and city centers compared to those living in villages.

In a study conducted in Iran, the eastern neighbor of Turkey, MS prevalence was found to be $34.7 \%$ according to ATP III, higher in females, in urban areas and in the 55-64 age group [24]. We found similar results. MS prevalence was $23.6 \%$ in Greece, $36.6 \%$ in Turkey and $34.7 \%$ in Iran. The low MS prevalence in Greece may be related to nutrition type and socio-economic level. Additionally, MS prevalence was found to be $27.7 \%$ in the Eastern Anatolian region of Turkey, which neighbors Iran. However, MS prevalences appear to differ between Eastern Anatolia and Iran. This difference may arise from different life styles (the fact that people are occupied with animal husbandry and have high physical activity rates may explain low MS prevalence) and consuming different foods. Differences

Table VI. Univariate and multivariate analysis of factors associated with metabolic syndrome

\begin{tabular}{|c|c|c|c|c|}
\hline Variables & $\begin{array}{l}\text { Univariate logistic regression } \\
\text { OR }(95 \% \mathrm{Cl})\end{array}$ & Value of $p$ & $\begin{array}{l}\text { Multiple logistic regression } \\
\text { OR }(95 \% \mathrm{Cl})\end{array}$ & Value of $p$ \\
\hline \multicolumn{5}{|l|}{ Gender } \\
\hline Male & 1 & & 1 & \\
\hline Female & $1.66(1.46-1.88)$ & $<0.001$ & $1.61(1.40-1.86)$ & $<0.001$ \\
\hline \multicolumn{5}{|l|}{ Age groups [years] } \\
\hline $18-25$ & 1 & & 1 & \\
\hline $26-30$ & $3.05(1.79-5.20)$ & $<0.001$ & $1.96(1.12-3.43)$ & 0.018 \\
\hline $31-35$ & $5.69(3.50-9.29)$ & $<0.001$ & $3.51(2.11-5.84)$ & $<0.001$ \\
\hline $36-40$ & $6.32(3.91-10.22)$ & $<0.001$ & $3.41(2.06-5.62)$ & $<0.001$ \\
\hline $41-45$ & $6.65(4.11-10.76)$ & $<0.001$ & $3.53(2.13-5.84)$ & $<0.001$ \\
\hline $46-50$ & $9.44(5.86-15.22)$ & $<0.001$ & $4.88(2.96-8.05)$ & $<0.001$ \\
\hline $51-55$ & $14.60(9.07-23.52)$ & $<0.001$ & $7.21(4.38-11.88)$ & $<0.001$ \\
\hline $56-60$ & $14.56(8.96-23.66)$ & $<0.001$ & $8.16(4.91-13.57)$ & $<0.001$ \\
\hline $61-65$ & $14.74(8.99-24.18)$ & $<0.001$ & $8.83(5.25-14.85)$ & $<0.001$ \\
\hline $66-70$ & $13.48(8.15-22.27)$ & $<0.001$ & $8.47(4.50-14.35)$ & $<0.001$ \\
\hline$\geq 71$ & $11.01(6.68-18.13)$ & $<0.001$ & $8.53(5.05-14.41)$ & $<0.001$ \\
\hline \multicolumn{5}{|l|}{ BMI } \\
\hline Normal & 1 & & 1 & \\
\hline Overweight & $3.40(2.77-4.17)$ & $<0.001$ & $2.74(2.22-3.39)$ & $<0.001$ \\
\hline Obese & $10.33(8.44-12.66)$ & $<0.001$ & $7.80(6.29-9.66)$ & $<0.001$ \\
\hline \multicolumn{5}{|l|}{ Altitude } \\
\hline Coastal & 1 & & 1 & \\
\hline Moderate elevation & $0.60(0.51-0.71)$ & $<0.001$ & $0.86(0.72-1.04)$ & 0.117 \\
\hline High elevation & $0.90(0.78-1.04)$ & 0.153 & $1.17(0.99-1.38)$ & 0.063 \\
\hline \multicolumn{5}{|l|}{ Location } \\
\hline City center & 1 & & 1 & \\
\hline District center & $1.08(0.94-1.24)$ & 0.299 & $0.99(0.85-1.16)$ & 0.900 \\
\hline Village & $0.58(0.69-0.96)$ & 0.015 & $0.78(0.65-0.94)$ & 0.010 \\
\hline
\end{tabular}


Table VII. Metabolic syndrome prevalence in provinces according to ATP III and IDF criteria

\begin{tabular}{|lccccccccc|}
\hline Provinces & \multicolumn{2}{c}{ Metabolic syndrome } & Provinces & \multicolumn{2}{c}{ Metabolic syndrome } & Provinces & \multicolumn{2}{c|}{ Metabolic syndrome } \\
\cline { 2 - 3 } & ATP III [\%] & IDF [\%] & & ATP III [\%] & IDF [\%] & & & ATP III [\%] & IDF [\%] \\
\hline Adana & 20 & 34.8 & Erzurum & 28.1 & 33.7 & Kars & 29.7 & 29.7 \\
\hline Ankara & 41.5 & 48.4 & Eskisehir & 39.5 & 54 & Kayseri & 39.6 & 42.6 \\
\hline Antalya & 36 & 45.1 & Gaziantep & 28 & 32.6 & Konya & 53.8 & 62 \\
\hline Bursa & 56 & 64 & Giresun & 27.4 & 33.1 & Samsun & 30.7 & 35.4 \\
\hline Canakkale & 52.8 & 57.1 & Hatay & 47.5 & 53.3 & Sivas & 41.1 & 46.8 \\
\hline Denizli & 32.3 & 43 & Istanbul & 51 & 63 & Sanliurfa & 22.5 & 26.9 \\
\hline Diyarbakir & 24.3 & 27.2 & Izmir & 46 & 52.5 & Van & 26.4 & 32.2 \\
\hline Edirne & 33.5 & 46.8 & Mersin & 23.2 & 29.8 & Zonguldak & 27 & 40.2 \\
\hline
\end{tabular}

among populations in different parts of the world in factors such as life style, physical activities, food culture, ethnic and genetic factors [21, 25-27] affect MS development [28-32].

Urbanization and decreased physical activity may lead to high MS prevalence [33, 34]. Individuals who live in rural areas are more physically active as they work in agriculture and animal husbandry and the low MS prevalences may be related to this [35]. In the study of Erem et al. carried out in Trabzon, in the Black Sea region in the northern part of Turkey, MS prevalence was detected as $26.9 \%$, hypertriglyceridemia as $30.4 \%$ and low HDL-C as $21.1 \%$ according to ATP III criteria [20,36]. Similarly, in this study, MS prevalence was found to be $28.4 \%$, hypertriglyceridemia $30.8 \%$ and low HDL-C $26.0 \%$ in the Black Sea region, and these rates are under the mean values for Turkey. The reason for this situation may be that the people who live in the Black Sea region are more physically active due to the geographic nature of the region (scarped and sloping) and consume a lot of anchovies, hazelnuts and vegetables. Metabolic syndrome prevalence was found to be highest in the Marmara region of Turkey according to both ATP III and IDF criteria. The highest MS rate was detected in Bursa among the 24 provinces according to both ATP III and IDF criteria. Urbanization, socio-economic level and national income are higher in the Marmara region, in the northwest part of Turkey, compared to other regions. Bursa is located in the Marmara region.

According to the 2009 data of the Turkish Statistics Institution (TSI), income per capita was found to be highest in the Marmara region [37]. The reason for high MS incidence may be urbanization, nutrition type and inadequate physical activity (sedentary life) [21, 25, 27, 33].

Abdominal obesity measures, one of the MS diagnostic criteria, vary by region and race. It is suggested to use IDF diagnostic criteria for Mediterranean populations [38]. IDF criteria were adopted since Turkey is a Mediterranean country.
According to IDF criteria, MS prevalence was found to be $17.9-42 \%$ in different studies conducted in Turkey $[14,39,40]$. In the aforementioned study of Sanisoglu et al., waist circumference was not measured, and obesity was used instead (BMI $>30 \mathrm{~kg} / \mathrm{m}^{2}$ ). Our previous study was carried out in the Mediterranean region in the southern part of Turkey. Similar results to ours were obtained from the study of Can et al., which was carried out in Istanbul (in the western part of Turkey) and Kayseri (in the central Anatolian region of Turkey) [40].

In our study, the reason for finding a higher MS prevalence according to IDF criteria may be due to our taking lower values as the criteria for fasting blood glucose and waist circumference both in males and females.

In the studies conducted in the neighbors of Turkey and in other countries, MS prevalence was detected as $32.1-43.4 \%$ according to IDF [1, 41-43].

When the rates of MS components were analyzed, HT was found to be the most prevalent according to both ATP III and IDF. In our previous study in the Mediterranean region, abdominal obesity was found to be the most prevalent component (88.7\%) [14]. The reason for higher rates of abdominal obesity in the Mediterranean region may be the high frequency of obesity in that region (43\%) [44]. In the study of Erem et al. from Trabzon in Turkey, HT was found to be the most prevalent component, with a frequency of $91.9 \%$ [45].

When the risk factors affecting MS were evaluated according to multiple logistic regression analysis in our study, female gender, age and obesity were found to be independent risk factors for MS. No published data are available in the literature investigating the aforementioned risk factors. In many studies carried out in Turkey and in the world, data are available indicating that MS prevalence increases with increasing BMI [15, 20, 46].

Living areas are classified as city center, district and village in Turkey. Metabolic syndrome risk was found to be lower in individuals living in villages 
according to univariate logistic regression analysis. Reduced physical activity due to urbanization may lead to high MS prevalence [33, 34]. Villagers are more physically active as they work in agriculture and animal husbandry and thus MS risk may be lower [35].

The limitations of our study are that cardiovascular risk was not clarified and long-term followup of the patients was not performed.

In conclusion, MS prevalence was found to be high in Turkey according to both ATP III and IDF criteria. It is necessary to work together with the national health system and other health financing and policy making institutions in Turkey to successfully diagnose and treat MS and prevent cardiovascular complications.

\section{References}

1. Ford ES. Prevalence of the metabolic syndrome defined by the International Diabetes Federation among adults in the U.S. Diabetes Care 2005; 28: 2745-9.

2. Laaksonen DE, Lakka HM, Niskanen LK, et al. Metabolic syndrome and development of diabetes mellitus: application and validation of recently suggested definitions of the metabolic syndrome in a prospective cohort study. Am J Epidemiol 2002; 156: 1070-7.

3. Miranda PJ, DeFronzo RA, Califf RM, Guyton JR. Metabolic syndrome: definition, pathophysiology, and mechanisms. Am Heart J 2005; 149: 33-45.

4. Grundy SM, Cleeman JI, Daniels SR, et al. American Heart Association; National Heart, Lung, and Blood Institute. Diagnosis and management of the metabolic syndrome: an American Heart Association/National Heart, Lung, and Blood Institute Scientific Statement. Circulation 2005; 112: 2735-52.

5. Wu SH, Liu Z, Ho SC. Metabolic syndrome and all-cause mortality: a meta-analysis of prospective cohort studies. Eur J Epidemiol 2010; 25: 375-84.

6. Sarrafzadegan N, Kelishadi R, Baghaei A, et al. Metabolic syndrome: an emerging public health problem in Iranian women: Isfahan Healthy Heart Program. Int J Cardiol 2008; 131: 90-6.

7. Alberti KG, Zimmet P, Shaw J. IDF Epidemiology Task Force Consensus Group. The metabolic syndrome: a new worldwide definition. Lancet 2005; 366: 1059-62.

8. Seneff S, Wainwright G, Mascitelli L. Is the metabolic syndrome caused by a high fructose, and relatively low fat, low cholesterol diet? Arch Med Sci 2011; 7: 8-20.

9. Athyros VG, Giouleme O, Ganotakis ES, et al. Safety and impact on cardiovascular events of long-term multifactorial treatment in patients with metabolic syndrome and abnormal liver function tests: a post hoc analysis of the randomised ATTEMPT study. Arch Med Sci 2011; 7: 796-805.

10. Zhu S, St-Onge MP, Heshka S, Heymsfield SB. Lifestyle behaviors associated with lower risk of having the metabolic syndrome. Metabolism 2004; 53: 1503-11.

11. Resnick HE. Strong Heart Study Investigators. Metabolic syndrome in American Indians. Diabetes Care 2002; 25: 1246-7.

12. Balkau B. Smoking, type 2 diabetes and metabolic syndrome. Diabetes Metab 2004; 30: 110-1.

13. Gupta A, Gupta R, Sarna M, et al. Prevalence of diabetes, impaired fasting glucose and insulin resistance syndrome in an urban Indian population. Diabetes Res Clin Pract 2003; 61: 69-76.

14. Gündogan K, Bayram F, Capak M, et al. Prevalence of metabolic syndrome in the Mediterranean region of Turkey: evaluation of hypertension, diabetes mellitus, obesity, and dyslipidemia. Metab Syndr Relat Disord 2009; 7: 427-34.

15. Onat A, Ceyhan K, Bașar O, et al. Metabolic syndrome: major impact on coronary risk in a population with low cholesterol levels: a prospective and cross-sectional evaluation. Atherosclerosis 2002; 165: 285-92.

16. Ozsahin AK, Gokcel A, Sezgin N, et al. Prevalence of the metabolic syndrome in a Turkish adult population. Diabetes Nutr Metab 2004; 17: 230-4.

17. Demiral Y, Soysal A, Can Bilgin A, et al. The association of job strain with coronary heart disease and metabolic syndrome in municipal workers in Turkey. J Occup Health 2006; 48: 332-8.

18. Soysal A, Demiral Y, Soysal D, et al. The prevalence of metabolic syndrome among young adults in Izmir, Turkey. Anadolu Kardiyol Derg 2005; 5: 196-201.

19. Eckel RH, Grundy SM, Zimmet PZ. The metabolic syndrome. Lancet 2005; 365: 1415-28.

20. Erem C, Hacihasanoglu A, Deger O, et al. Prevalence of metabolic syndrome and associated risk factors among Turkish adults: Trabzon MetS study. Endocrine 2008; 33: 9-20.

21. Panagiotakos DB, Pitsavos C, Chrysohoou C, et al. Impact of lifestyle habits on the prevalence of the metabolic syndrome among Greek adults from the ATTICA study. Am Heart J 2004; 147: 106-12.

22. Cankurtaran M, Halil M, Yavuz BB, Dagli N, Oyan B, Ariogul S. Prevalence and correlates of metabolic syndrome (MS) in older adults. Arch Gerontol Geriatr 2006; 42: 35-45.

23. Athyros VG, Bouloukos VI, Pehlivanidis AN, et al. MetSGreece Collaborative Group. The prevalence of the metabolic syndrome in Greece: the MetS-Greece Multicentre Study. Diabetes Obes Metab 2005; 7: 397-405.

24. Delavari A, Forouzanfar MH, Alikhani S, Sharifian A, Kelishadi R. First nationwide study of the prevalence of the metabolic syndrome and optimal cutoff points of waist circumference in the Middle East: the national survey of risk factors for noncommunicable diseases of Iran. Diabetes Care 2009; 32: 1092-7.

25. O'Neil CE, Fulgoni VL 3rd, Nicklas TA. Candy consumption was not associated with body weight measures, risk factors for cardiovascular disease, or metabolic syndrome in US adults: NHANES 1999-2004. Nutr Res 2011; 31: 122-30.

26. Ozsait B, Kömürcü Bayrak E, Poda M, et al. CETP TaqIB polymorphism in Turkish adults: association with dyslipidemia and metabolic syndrome. Anadolu Kardiyol Derg 2008; 8: 324-30

27. Kraja AT, Rao DC, Weder AB, et al. An evaluation of the metabolic syndrome in a large multi-ethnic study: the Family Blood Pressure Program. Nutr Metab (Lond) 2005; 2: 17.

28. Scholze J, Alegria E, Ferri C, et al. Epidemiological and economic burden of metabolic syndrome and its consequences in patients with hypertension in Germany, Spain and Italy: a prevalence-based model. BMC Public Health 2010; 10: 529.

29. Sidorenkov O, Nilssen O, Grjibovski AM. Metabolic syndrome in Russian adults: associated factors and mortality from cardiovascular diseases and all causes. BMC Public Health 2010; 10: 582.

30. Al Suwaidi J, Zubaid M, El-Menyar AA, et al. Prevalence of the metabolic syndrome in patients with acute co- 
ronary syndrome in six Middle Eastern Countries. J Clin Hypertens (Greenwich) 2010; 12: 890-9.

31. Saito I, Mori M, Shibata H, Hirose H, Tsujioka M, Kawabe H. Prevalence of metabolic syndrome in young men in Japan. J Atheroscler Thromb 2007; 14: 27-30.

32. Wang L, Tao Y, Xie Z, et al. Prevalence of metabolic syndrome, insulin resistance, impaired fasting blood glucose, and dyslipidemia in Uygur and Kazak populations. J Clin Hypertens (Greenwich) 2010; 12: 741-5.

33. Shuval K, DeVahl J, Tong L, Gimpel N, Lee JJ, DeHaven MJ. Anthropometric measures, presence of metabolic syndrome, and adherence to physical activity guidelines among African American church members, Dallas, Texas, 2008. Prev Chronic Dis 2011; 8: A18.

34. du Plessis A, Malan L, Malan NT. Coping and metabolic syndrome indicators in urban black South African men: the SABPA study. Cardiovasc J Afr 2010; 21: 268-73.

35. Sobngwi E, Mbanya JC, Unwin NC, et al. Physical activity and its relationship with obesity, hypertension and diabetes in urban and rural Cameroon. Int I Obes Relat Metab Disord 2002; 26: 1009-16.

36. Erem C, Hacihasanoglu A, Deger O, Kocak M, Topbas M. Prevalence of dyslipidemia and associated risk factors among Turkish adults: Trabzon lipid study. Endocrine 2008; 34: $36-51$.

37. 25.TURKSTAT, Income and Living Conditions Survey, 2002009.

38. Alberti KG, Eckel RH, Grundy SM, et al. International Diabetes Federation Task Force on Epidemiology and Prevention; Hational Heart, Lung, and Blood Institute; American Heart Association; World Heart Federation; International Atherosclerosis Society; International Association for the Study of Obesity. Harmonizing the metabolic syndrome: a joint interim statement of the International Diabetes Federation Task Force on Epidemiology and Prevention; National Heart, Lung, and Blood Institute; American Heart Association; World Heart Federation; International Atherosclerosis Society; and International Association for the Study of Obesity. Circulation 2009; 120: 1640-5.

39. Sanisoglu SY, Oktenli C, Hasimi A, Yokusoglu M, Ugurlu M. Prevalence of metabolic syndrome-related disorders in a large adult population in Turkey. BMC Public Health 2006; 6: 92.

40. Can AS, Bersot TP. Analysis of agreement among definitions of metabolic syndrome in nondiabetic Turkish adults: a methodological study. BMC Public Health 2007; 7: 353 .

41. Zabetian A, Hadaegh F, Azizi F. Prevalence of metabolic syndrome in Iranian adult population, concordance between the IDF with the ATPIII and the WHO definitions. Diabetes Res Clin Pract 2007; 77: 251-7.

42. Athyros VG, Ganotakis ES, Elisaf M, Mikhailidis DP. The prevalence of the metabolic syndrome using the National Cholesterol Educational Program and International Diabetes Federation definitions. Curr Med Res Opin 2005; 21: 1157-9.

43. Moy FM, Bulgiba A. The modified NCEP ATP III criteria maybe better than the IDF criteria in diagnosing Metabolic Syndrome among Malays in Kuala Lumpur. BMC Public Health 2010; 10: 678.

44. Gokcel A, Ozsahin AK, Sezgin N, et al. High prevalence of diabetes in Adana, a southern province of Turkey. Diabetes Care 2003; 26: 3031-4.

45. Erem C, Arslan C, Hacihasanoglu A, et al. Prevalence of obesity and associated risk factors in a Turkish population (Trabzon city, Turkey). Obes Res 2004; 12: 1117-27.
46. Jaber LA, Brown MB, Hammad A, Zhu Q, Herman WH. The prevalence of the metabolic syndrome among Arab Americans. Diabetes Care 2004; 27: 234-8. 